\title{
Morbidity in 47,XYY syndrome: a nationwide epidemiological study of hospital diagnoses and medication use
}

\author{
Agnethe Berglund, PhD ${ }^{1,2,3}$, Kirstine Stochholm, PhD, DMSc ${ }^{3}{ }^{3}$ and \\ Claus Højbjerg Gravholt, Professor (iD ${ }^{2,3}$
}

\begin{abstract}
Purpose: A systematic description of morbidity in 47,XYY syndrome based on nationwide registry data of hospital diagnoses and prescribed medication.
\end{abstract}

Methods: All males in Denmark diagnosed with 47,XYY syndrome during 1960-2014 were identified. Each was matched with 100 male controls from the general population. Diagnoses related to hospital encounters (1977-2014) and prescriptions (1996-2014) were analyzed by negative binominal regression and Cox regression, respectively.

Results: 47,XYY syndrome was associated with a significantly increased overall incidence of hospital diagnoses (incidence rate ratio $=2.30$, confidence interval $[\mathrm{CI}]: 1.99-2.65$ ), including a significantly increased incidence of diagnoses associated with congenital malformations and genetic disorders as well as with psychiatric, neurologic, respiratory, urogenital, endocrine, circulatory, gastrointestinal, and musculoskeletal system disorders. Diagnoses associated with infections, skin and eye disorders were significantly increased as well. 47,XYY syndrome was associated with a significantly increased occurrence of prescriptions overall (hazard ratio $=1.25, \mathrm{CI}: 1.10-1.44$ ), with sex hormones and medication related to the urogenital system, blood, and nervous system being most prominently increased.

Conclusion: 47,XYY syndrome is associated with a significantly increased morbidity owing to a wide variety of diseases. Increased awareness of the diverse morbidity in 47,XYY syndrome may help guide clinicians assessing 47,XYY males, thereby improving longterm health outcomes.

Genetics in Medicine (2020) 22:1542-1551; https://doi.org/10.1038/s41436020-0837-y

Keywords: 47,XYY syndrome; morbidity; hospital diagnoses; medication; registry study

\section{INTRODUCTION}

The 47,XYY syndrome is a sex chromosome abnormality (SCA) in males caused by nondisjunction during paternal meiosis II or during postzygotic mitosis. Pooling data from a number of newborn chromosome surveys performed decades ago, the 47,XYY syndrome has been estimated to affect approximately 1 per 1000 male births, thus making 47,XYY one of the most common SCAs. ${ }^{1}$ However, failure to diagnose is extensive. Only $20 \%$ of the expected number of $47, \mathrm{XYY}$ males are being clinically ascertained. ${ }^{1}$ Further, diagnosis is often considerably delayed. The median age at diagnosis is 15 years, with some being diagnosed even very late in life. ${ }^{1}$

Although recognized since the 1960s, knowledge about the $47, \mathrm{XYY}$ phenotype is limited. Except for tall stature, readily explained by the presence of three copies of the SHOX gene located in the pseudoautosomal regions of the sex chromosomes, ${ }^{2}$ the clinical phenotype seems highly variable and affected individuals may not present physical features to a degree that distinguishes them from the normal male population. One of the first studies reporting on the $47, \mathrm{XYY}$ syndrome found an increased prevalence of males with a 47 , XYY karyotype among patients in a Scottish State Hospital for the mentally ill ${ }^{3}$ and subsequently, most studies have focused on describing the neurocognitive and psychopathological phenotype associated with the 47,XYY syndrome. An impaired neurocognitive profile with speech and language disorders, behavioral problems, as well as reduced motor skills and educational achievements has been reported. ${ }^{4-6}$ Knowledge about long-term health outcomes in males affected by the $47, \mathrm{XYY}$ syndrome is, however, extremely limited and primarily based on case reports or small selected cohorts. Exceptions are the few population-based studies from the UK and Denmark, which reported that the 47,XYY syndrome is associated with a significantly increased mortality owing to a variety of causes compared with the general population. $^{7,8}$

To add knowledge on associations between the 47,XYY syndrome and long-term health outcomes, we here perform a systematic and comprehensive epidemiological description of morbidity among males in the Danish $47, \mathrm{XYY}$ cohort using

${ }^{1}$ Department of Clinical Genetics, Aarhus University Hospital, Aarhus, Denmark; ${ }^{2}$ Department of Molecular Medicine, Aarhus University Hospital, Aarhus, Denmark; ${ }^{3}$ Department of Endocrinology and Internal Medicine, Aarhus University Hospital, Aarhus, Denmark. Correspondence: Agnethe Berglund (agnethe.berglund@clin.au.dk) 
Table 1 Median year of birth, median year of diagnosis, and median age at diagnosis within the entire 47,XYY cohort.

\begin{tabular}{lllll} 
& All 47,XYY & Nonmosaic 47,XYY & $46, X Y / 47, X Y Y$ & Other \\
\hline Number & 251 & 202 & 28 & 21 \\
Year of birth & $1976(1899-2013)$ & $1975(1909-2013)$ & $1981(1899-2008)$ & $1970(1910-2011)$ \\
Year of diagnosis & $1996(1965-2014)$ & $1994(1965-2014)$ & $1999(1968-2014)$ & $2003(1969-2013)$ \\
\hline Age at diagnosis & $17.5(0-70.7)$ & $17.3(0-68.1)$ & $9.0(0-70.7)$ & $28.8(0-66.5)$ \\
\hline
\end{tabular}

Ranges shown in parentheses.

longitudinally collected data on hospital diagnoses and prescribed medication.

\section{MATERIALS AND METHODS}

\section{Setting}

Denmark has a universal tax-funded health-care system with residency-based entitlement ensuring free and equal healthcare access for all residents.

\section{Cases and controls}

From the Danish Cytogenetic Central Registry (DCCR), all persons registered with $47, \mathrm{XYY}$ or compatible karyotypes during 1960-2014 were identified. Accepted karyotypes were $47, \mathrm{XYY}, 46, \mathrm{XY} / 47, \mathrm{XYY}$, and other $47, \mathrm{XYY}$ mosaic karyotypes. All cases were matched with 100 randomly selected agematched (month and year of birth) male controls from the Danish general population by the Division of Research Services at Statistics Denmark.

\section{Data sources}

Data regarding hospital diagnoses and prescribed medication were retrieved from the Danish National Patient Registry (DNPR) (1977-2014) and the Danish Prescription Registry (1996-2014). The Causes of Death Registry provided data regarding death (1977-2014). For more details, see Supplementary Material and Methods.

\section{Statistics}

Hospital diagnoses were analyzed by negative binomial regression yielding incidence rate ratios (IRRs) as the measure of association. The outcomes studied were (1) inpatient and outpatient hospital diagnoses regardless of diagnosis and (2) inpatient and outpatient diagnoses for (a) chapters according to the 10th edition of the International Classification of Diseases (ICD-10), (b) ICD-10 blocks, and (c) specific diagnoses. As the 47 , XYY syndrome is a congenital condition, time at risk started at birth and ended at death, emigration, or at end of follow-up (31 December 2014), whichever came first. Each stratum of a male with 47,XYY and his matched controls constituted a cluster, and this clustering was allowed using a random effects model.

Prescribed medication were analyzed using stratified Cox regression yielding hazard ratios (HRs) as the measure of association. Each 47,XYY male and his matched controls constituted a stratum. Time at risk started at birth. If born prior to 1 January 1995, entry was delayed until 1 January
1995. Time at risk ended at the event of interest, at death, at emigration, or at end of follow-up, whichever came first. The events of interest were (1) first prescription of any medication; and (2) first prescription of medication according to (a) chapters defined in accordance to the Anatomical Therapeutic Classification (ATC) system, (b) ATC blocks, and (c) specific medications. Specific diagnoses and specific medications were only analyzed in the case of a significant difference at a more aggregate level.

All analyses were performed using Stata software, version 14.1 (Stata Corp., College Station, TX). $P$ values $<0.05$ were considered to indicate statistically significant differences.

\section{Ethics}

The project was approved by the DCCR and the Danish Data Protection Authority (journal number 2013-41-2017). Data were accessed by a secure remote access to Statistics Denmark. To avoid any possibility of personal identification of cases, Statistics Denmark prohibits specification of the exact number of cases with a given condition if less than three, thus reported as $<3$.

\section{RESULTS}

A total of 255 individuals with 47,XYY or compatible karyotypes were identified. Of those, two were excluded because of an invalid identification number, as well as another two, who were officially registered as females and therefore matched with female controls by Statistics Denmark. Thereby, 251 cases were left for the analyses. Supplementary Table 1 provides information regarding distribution of karyotypes within the cohort. Data regarding year of birth, year of diagnosis, and age at diagnosis are provided in Table 1. In the following, " $47, \mathrm{XYY}$ " is used when referring to the entire cohort, unless otherwise specified.

\section{Hospital diagnoses}

The overall risk of hospital diagnoses was more than two times increased among 47,XYY males compared with controls $(\mathrm{IRR}=2.30$, CI:1.99-2.65), and 47,XYY males were significantly younger than controls when receiving their first hospital diagnosis $(P=0.0013)$ (Fig. 1). Stratified by karyotype, the overall risk of hospital diagnoses was significantly increased for nonmosaic $47, \mathrm{XYY}$ males (IRR $=2.43, \mathrm{CI}$ : 2.10-2.81) and for males with other karyotypes (IRR $=1.76$, CI: 1.10-2.82), whereas no significant difference was observed for males with $46, \mathrm{XY} / 47, \mathrm{XYY}$ mosaicism $(\mathrm{IRR}=1.82$, CI: 


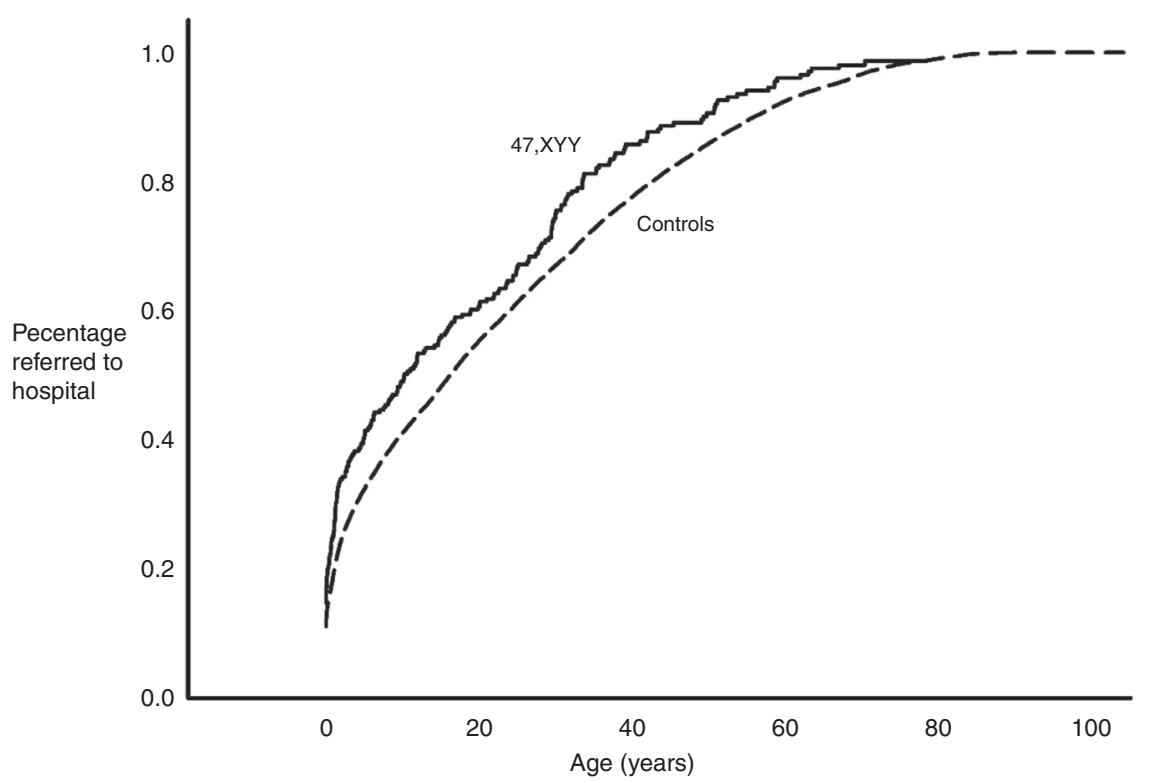

Fig. 1 Proportion of 47,XYY males and controls from the general male population being referred to hospital for the first time.

0.81-4.09). For the entire 47,XYY cohort, dividing diagnoses into chapters according to the ICD-10, a significant increased risk was observed for the following chapters: congenital malformations and genetic disorders (including 47,XYY), psychiatric disorders, neurological disorders, respiratory disorders, urogenital disorders, endocrine disorders, "other" disorders (symptoms and signs), circulatory system disorders, gastrointestinal disorders, skin diseases, infectious diseases, eye diseases, disorders related to the connective tissue and bones/musculoskeletal system, and disorders caused by external factors (14 of 18 chapters) (Fig. 2a). By further subdividing each chapter, it was possible to get a more detailed picture of the impact of certain diagnoses within chapters. Table 2 provides information about diagnoses occurring with either significantly increased or decreased frequency.

\section{Prescribed medication}

The overall risk of having any medication prescribed was significantly increased among 47,XYY males compared with controls $(\mathrm{HR}=1.25$, CI: $1.10-1.44)$. Stratified on karyotype, the risk was increased for nonmosaic 47,XYY males (HR = 1.37, CI: $1.18-1.60)$, whereas no significant difference was observed for males with $46, \mathrm{XY} / 47, \mathrm{XYY}$ mosaicism $(0.76$, CI: $0.50-1.15)$ and other karyotypes (HR $=1.28$, CI: 0.78-2.11). For the entire 47,XYY cohort, dividing prescribed medication into chapters according to the ATC system, a significant increased risk was observed for the following chapters: genitourinary system and sex hormones, blood and blood forming organs, nervous system, respiratory system, cardiovascular system, musculoskeletal system, systemic hormones -excluding sex hormones and insulins, alimentary tract and metabolism, dermatologicals, anti-infectives for systemic use, and sensory organs (11 of 14 chapters) (Fig. 2b). As for hospital diagnoses, prescribed medication were subdivided to investigate the impact of specific medications within each chapter. Table 3 provides information about medication found to be prescribed with either significantly increased or decreased frequency.

\section{DISCUSSION}

This systematic investigation of longitudinally collected data of hospital diagnoses and prescribed medication in a national cohort of males diagnosed with 47,XYY syndrome, appears to be the sole analysis of its kind. 47,XYY syndrome was associated with a significantly increased overall risk of hospital diagnoses as well with a significantly increased overall risk of being prescribed medication. Interpreted from the increased risk of hospital diagnoses in 14 of 18 diagnostic chapters as well as from the increased risk of prescriptions in 11 of 14 medication chapters, 47,XYY syndrome was associated with a significant increased morbidity that was not confined to a single or few diseases but indeed to a wide variety of diseases.

Testosterone levels in males with 47,XYY syndrome may range from significantly higher ${ }^{9}$ to similar ${ }^{10,11}$ or significantly lower ${ }^{10}$ than in controls. In the present study, 47,XYY syndrome was associated with an increased risk of gonadal dysfunction along with an increased risk of having androgens prescribed. Testosterone has a major influence on metabolism, and testosterone deficiency is associated with a negative altered metabolic profile leading to an unfavorable body composition with increased visceral fat and decreased muscle mass. Here, a diagnosis of obesity was observed in considerably more $47, \mathrm{XYY}$ males $(n=22)$ than was gonadal dysfunction $(n=3)$. In addition, 47,XYY males had a significant increased risk of obesity compared with controls. Owing to the bidirectional relationship of hypogonadism and obesity, it can be speculated whether part of those diagnosed with obesity are suffering from an underlying hypogonadism, 
a

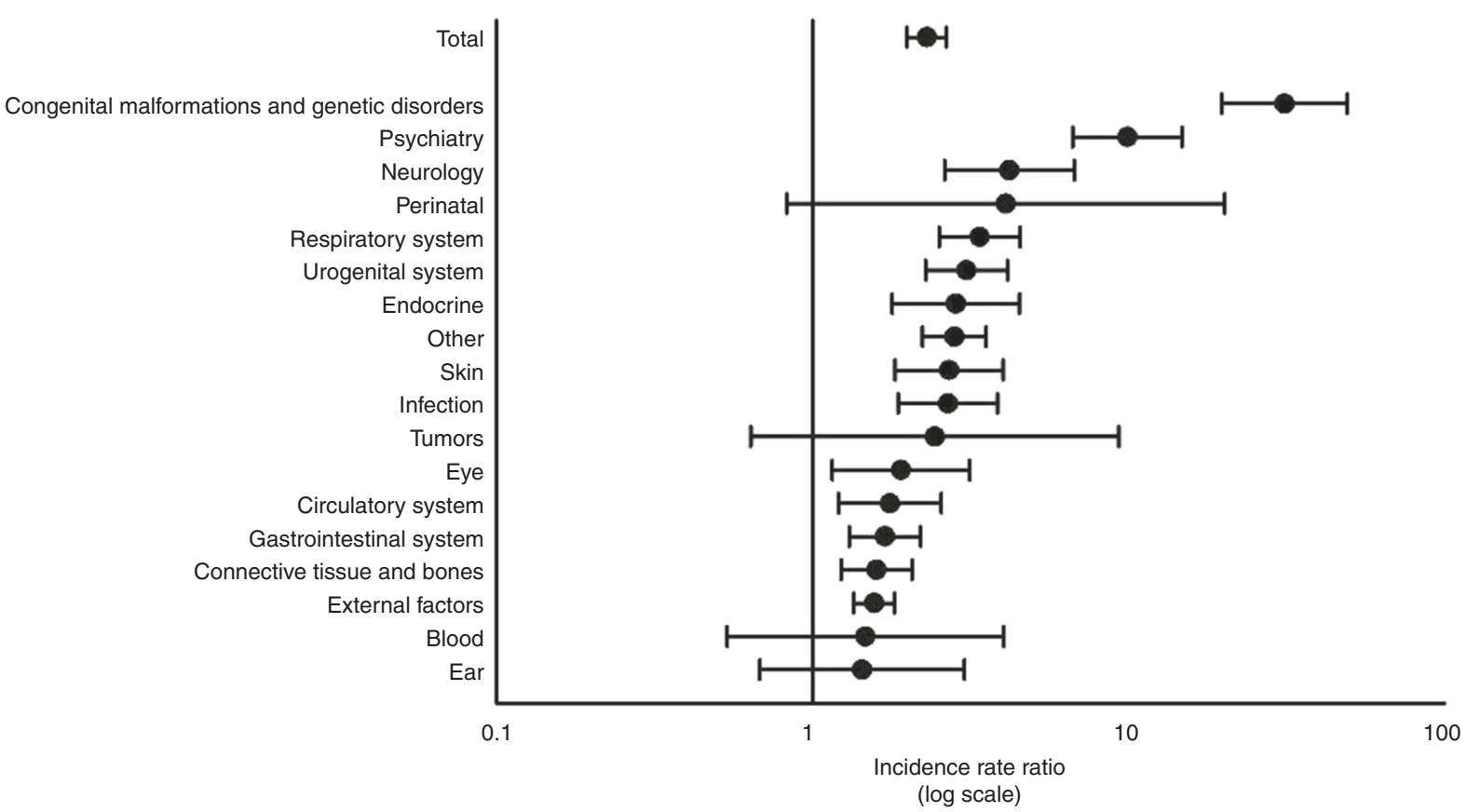

b

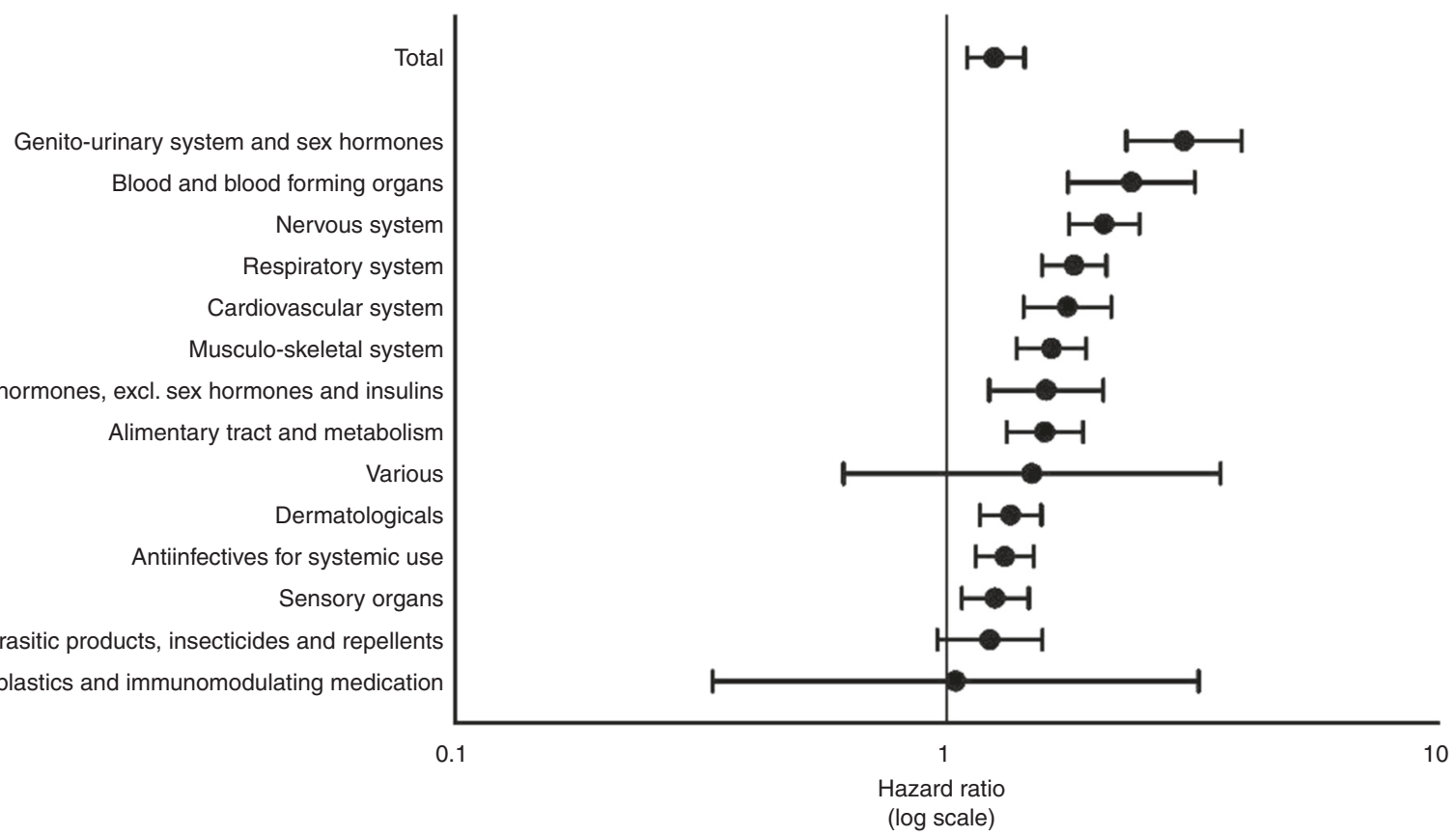

Fig. 2 Comparison of hospital diagnoses and prescribed medication among 47,XYY males and general population male controls. (a) Diagnoses are divided in diagnostic chapters according to the International Classification of Diseases, 10th edition. (b) Prescribed medication is divided in chapters according to the Anatomic Therapeutic Classification system.

thus indeed making hypogonadism more prevalent in 47,XYY syndrome than when solely interpreted from the diagnoses of gonadal dysfunction and androgen prescriptions. The increased occurrence of both gynecomastia, osteoporosis, and fractures, as well as of prescriptions for erectile dysfunction among 47,XYY males further substantiates an increased risk of hypogonadism among these males. Hitherto, hypogonadism has only been firmly linked to Klinefelter syndrome. ${ }^{12}$ Future studies should focus on investigating gonadal function in $47, \mathrm{XYY}$ syndrome by including data on circulating testosterone and gonadotropin levels. Type 2 diabetes, hyperlipidemia, hypertension, all seen more frequently in Klinefelter syndrome, ${ }^{13}$ and ischemic heart disease are long-term consequences of hypogonadism or of obesity itself. ${ }^{14}$ However, none of these were found to occur with increased frequency among 47,XYY males. Surprisingly, there was a significantly reduced occurrence of hyperlipidemia as well as of ischemic heart disease, the latter being in contrast to 
Table 2 Hospital diagnoses among 47,XYY males and general population male controls.

\begin{tabular}{|c|c|c|c|c|c|}
\hline Diagnosis & ICD-10 code & ICD-8 code & $\begin{array}{l}47, X Y Y \\
(n)\end{array}$ & $\begin{array}{l}\text { Controls } \\
(n)\end{array}$ & $\begin{array}{l}\text { Incidence rate ratio } \\
(95 \% \mathrm{Cl})\end{array}$ \\
\hline Infections & A00-B99 & 000-136 & 60 & 3715 & $2.68(1.87-3.86)$ \\
\hline Neoplasms & C00-D48 & $140-239$ & 21 & 1898 & $2.44(0.64-9.32)$ \\
\hline Blood & D50-D89 & 280-289 & 9 & 836 & $1.47(0.54-4.03)$ \\
\hline Endocrinology & E00-E90 & 240-279 & 55 & 2683 & $2.84(1.79-4.51)$ \\
\hline Acromegaly & E220 & 25300,25301 & 3 & 5 & $8.33(1.47-47.31)$ \\
\hline Obesity & E66 & 27799 & 22 & 456 & $5.49(2.79-10.77)$ \\
\hline Gonadal dysfunction & E29 & $25709,25719,25799$ & 3 & 20 & $58.56(7.03-488.09)$ \\
\hline Testicular hypofunction & E291 & 25719 & 3 & 16 & $79.18(8.78-714.12)$ \\
\hline Hyperlipidemia & E780-E785 & $27200-27201$ & 3 & 725 & $0.33(0.11-0.98)$ \\
\hline Psychiatry & FO0-F99 & $290-315$ & 84 & 2615 & $9.92(6.65-14.78)$ \\
\hline $\begin{array}{l}\text { Mental or behavioral disorders due to use of alcohol } \\
\text { or drugs }\end{array}$ & F10-F19 & $3030-304$ & 33 & 1535 & $4.30(2.31-8.02)$ \\
\hline Schizophrenia & $\mathrm{F} 20$ & 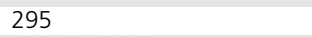 & 5 & 99 & $19.41(4.36-86.38)$ \\
\hline Bipolar & F31 & 29619-29699 & 4 & 36 & $38.38(5.93-248.32)$ \\
\hline Reaction to stress and adjustment disorders & F43 & 30802-30806 & 6 & 172 & $13.93(2.96-65.66)$ \\
\hline Personality disorder & F60-F62, F69 & 301 & 3 & 65 & $6.72(1.29-35.13)$ \\
\hline Disorders of gender identity and sexual preference & F64-F68 & 302 & 4 & 5 & $3748.27(5.62-2501362)$ \\
\hline Mental retardation & F70-F79 & $310-315$ & 18 & 104 & $432.32(136.47-1369.54)$ \\
\hline $\begin{array}{l}\text { Disorders of psychological development, } \\
\text { including autism }\end{array}$ & F80-89 & 29900-29903 & 15 & 95 & $319.37(94.46-1079.86)$ \\
\hline Neurology & G00-G99 & $320-358$ & 53 & 2693 & $4.20(2.62-6.73)$ \\
\hline Epilepsy & G40-G41 & 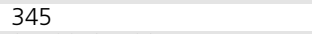 & 25 & 605 & $10.71(4.79-23.96)$ \\
\hline Sleep disorders & G47 & 34700-34709 & 9 & 360 & $3.11(1.38-7.04)$ \\
\hline Sleep apnea & G473 & NA & 9 & 307 & $3.12(1.42-6.87)$ \\
\hline Ophthalmology & H0O-H59 & 360-379 & 32 & 2633 & $1.91(1.16-3.14)$ \\
\hline Glaucoma & $\mathrm{H} 40-\mathrm{H} 42$ & 375 & 6 & 99 & $18.05(3.49-93.44)$ \\
\hline Myopia & H521 & 37002 & 7 & 109 & $7.73(1.20-49.87)$ \\
\hline Astigmatism & H523 & 37000 & 3 & 54 & $9.39(1.57-56.26)$ \\
\hline Otology & H60-H95 & $380-389$ & 27 & 2518 & $1.44(0.68-3.03)$ \\
\hline Circulatory system diseases & $100-199$ & $390-458$ & 65 & 4891 & $1.76(1.21-2.56)$ \\
\hline Ischemic heart disease & $120-125$ & $410-414$ & 13 & 1547 & $0.43(0.23-0.82)$ \\
\hline Venous thromboembolism and thrombophlebitis & $181-182$ & $451-453$ & 17 & 355 & $10.25(4.65-22.60)$ \\
\hline Varicose veins of lower extremities & 183 & 454 & 17 & 432 & $12.70(5.99-26.94)$ \\
\hline Respiratory system & J00-J99 & 460-519 & 117 & 7077 & $3.38(2.51-4.54)$ \\
\hline Acute respiratory infections & $\mathrm{J} 00-\mathrm{J} 22$ & $460-486$ & 72 & 3774 & $4.12(2.90-5.85)$ \\
\hline Pneumonia & J12-J18 & $480-486$ & 54 & 2045 & $5.49(3.72-8.10)$ \\
\hline Acute lower respiratory infection, other & $J 20-J 22$ & 466 & 28 & 705 & $12.52(6.23-25.16)$ \\
\hline Chronic lower respiratory diseases & $\mathrm{J} 440-\mathrm{J} 47$ & 490-493 & 51 & 1784 & $5.90(3.65-9.54)$ \\
\hline Chronic obstructive lung disease & j44 & & 9 & 501 & $5.79(2.42-15.15)$ \\
\hline Asthma & $\int 45-J 47$ & 493 & 43 & 1180 & $5.88(3.38-10.22)$ \\
\hline Oral cavity and gastrointestinal disorders & K00-K93 & 520-577 & 99 & 7355 & $1.69(1.31-2.20)$ \\
\hline Disorders of tooth development and eruption & K00 & 520 & 6 & 155 & $7.02(2.01-24.46)$ \\
\hline Caries and other diseases of hard tissues of teeth & K02-K03 & 521 & 10 & 237 & $3.96(1.52-10.37)$ \\
\hline Anomalies of jaw and tooth position & K07 & 524 & 5 & 172 & $3.91(1.16-13.12)$ \\
\hline Fibrosis and cirrhosis of liver & K74 & 571 & 3 & 114 & $7.83(1.23-49.77)$ \\
\hline Gastrointestinal hemorrhage & K920-K922 & $56915,78459,78579$ & 8 & 283 & $4.06(1.64-10.08)$ \\
\hline Skin & L00-L99 & $680-709$ & 54 & 3163 & $2.71(1.83-4.01)$ \\
\hline Skin infections & LO0-L08 & $680-686$ & 29 & 1728 & $1.95(1.19-3.21)$ \\
\hline Dermatitis and eczema & L20-L30 & $690-693$ & 12 & 515 & $4.19(1.53-11.47)$ \\
\hline Ulcer of lower limb and unspecified ulcer of skin & L97, L984 & 707 & 8 & 126 & $27.50(7.36-102.57)$ \\
\hline Connective tissue and bones & M00-M99 & 710-738 & 97 & 7680 & $1.59(1.23-2.06)$ \\
\hline Valgus & M210 & 73802 & $<3$ & 4 & $2526.99(96.02-66503.84)$ \\
\hline Flat foot & M214 & 73699 & 4 & 51 & $33.45(7.57-147.83)$ \\
\hline Back pain & M54 & $71709,72809,72859,72899$ & 23 & 1104 & $2.42(1.49-3.94)$ \\
\hline Osteoporosis & M80-M82 & 72309 & 7 & 153 & $5.56(1.66-18.56)$ \\
\hline Urogenital system & N00-N99 & $580-629$ & 90 & 4537 & $3.07(2.28-4.14)$ \\
\hline Nephrolithiasis & $\mathrm{N} 20-\mathrm{N} 22$ & 592,594 & 13 & 518 & $6.52(3.11-13.64)$ \\
\hline Male genital diseases & N40-N45, N47-N51 & $600-605,607-608$ & 28 & 1880 & $1.59(1.03-2.46)$ \\
\hline Gynecomastia & N629 & 61111 & 6 & 199 & $7.52(2.73-20.73)$ \\
\hline Infertility & N46 & 60699 & 29 & 399 & $11.27(6.00-21.15)$ \\
\hline Perinatal disease & P00-P96 & $760-779$ & 34 & 2572 & $4.09(0.83-20.15)$ \\
\hline Congenital malformations & Q00-Q99 & $740-759$ & 97 & 1996 & 31.15 (19.73-49.17) \\
\hline Circulatory system malformations & Q20-Q28 & $746-748$ & 9 & 213 & $59.3(7.63-460.54)$ \\
\hline Genitalia and gonads & Q50-Q56 & $752-1$ & 16 & 686 & $3.45(1.65-7.24)$ \\
\hline Cryptorchidism & Q53 & $75210-75211,75219$ & 16 & 501 & $4.88(2.25-10.56)$ \\
\hline Musculoskeletal system & Q65-Q79 & $754-756$ & 18 & 472 & $10.86(4.24-27.83)$ \\
\hline Malformations of the feet & Q66 & 754 & 9 & 168 & $29.76(5.55-159.58)$ \\
\hline Other diagnoses & R00-R99 & 780-796 & 132 & 8613 & $2.81(2.23-3.55)$ \\
\hline Febrile convulsions & R560 & 78021 & 13 & 501 & $3.30(1.48-7.37)$ \\
\hline Syncope and collapse & R55 & $78250-78259$ & 19 & 784 & $3.51(2.01-6.14)$ \\
\hline External & S00-T98; V00-Y09 & 800-999; E8000-E9999 & 202 & 19666 & $1.57(1.35-1.82)$ \\
\hline All fractures & $\begin{array}{l}\mathrm{S} 02, \mathrm{~S} 12, \mathrm{~S} 22, \mathrm{S32}, \\
\mathrm{S} 42, \mathrm{~S} 52, \mathrm{~S} 62, \mathrm{~s} 2, \\
\mathrm{~S} 82, \mathrm{~s} 92, \mathrm{~T}\end{array}$ & $800-829$ & 98 & 8482 & $1.48(1.18-1.87)$ \\
\hline Potential osteoporotic fractures & $\begin{array}{l}\text { S120-S127, S320, S52, } \\
\text { S720-S722 }\end{array}$ & $805,813,820$ & 11 & 737 & $2.12(1.08-4.19)$ \\
\hline
\end{tabular}

Incidence rate ratios (IRRs) for chapters according to the 10th edition of the International Classification of Diseases (ICD-10), whether significant or not, are shown in bold. If significant, IRRs of ICD-10 blocks and specific diagnoses (italic) within the chapters are provided. According to ethics regulations, the exact number of cases with a given diagnosis is not provided if less than four.

Cl confidence interval. 
Table 3 Prescribed medication among 47,XYY males and general population male controls.

\begin{tabular}{|c|c|c|c|c|}
\hline Medication & ATC code & $47, \mathrm{XYY}(n)$ & Controls $(n)$ & Hazard ratio $(95 \% \mathrm{Cl})$ \\
\hline Alimentary tract and metabolism & A & 118 & 10,713 & $1.51(1.26-1.81)$ \\
\hline Stomatologica & A01 & 42 & 2950 & $1.59(1.17-2.16)$ \\
\hline Caries prophylactic agents & $A 01 A A$ & 17 & 471 & $4.62(2.84-7.51)$ \\
\hline Acid-related disorders & A02 & 70 & 5906 & $1.78(1.41-2.26)$ \\
\hline Antacids & $A 02 A$ & 21 & 793 & $4.46(2.87-6.690)$ \\
\hline Peptic ulcer and reflux & $A 02 B$ & 65 & 5657 & $1.66(1.30-2.12)$ \\
\hline Constipation & A06 & 22 & 1541 & $2.49(1.63-3.80)$ \\
\hline Antiobesity preparations & A08 & 12 & 627 & $2.32(1.31-4.11)$ \\
\hline Blood and blood forming organs & B & 41 & 3457 & $2.18(1.60-2.98)$ \\
\hline Antithrombotic agents & B01 & 30 & 2796 & $1.97(1.37-2.83)$ \\
\hline Antianemic preparations & B03 & 19 & 1037 & $3.19(2.02-5.05)$ \\
\hline Iron & $B 03 A$ & 8 & 346 & $4.59(2.25-9.34)$ \\
\hline B12 and folic acid & $B 03 B$ & 12 & 775 & $2.44(1.38-4.33)$ \\
\hline Cardiovascular system & C & 87 & 8497 & $1.66(1.34-2.05)$ \\
\hline Heart therapy & C01 & 21 & 1456 & $2.81(1.82-4.35)$ \\
\hline Cardiac glycosides & C01A & 7 & 412 & $4.46(2.08-9.55)$ \\
\hline Cardiac stimulant excluding cardiac glycosides & $\operatorname{cotc}$ & 5 & 156 & $4.13(1.69-10.11)$ \\
\hline Vasodilators used in cardiac disease & CO1D & 12 & 954 & $2.34(1.32-4.15)$ \\
\hline Diuretics & $\mathrm{CO3}$ & 33 & 2668 & $2.52(1.78-3.56)$ \\
\hline Vasoprotectives & $\mathrm{C06}$ & 50 & 4081 & $1.60(1.21-2.12)$ \\
\hline Dermatologicals & D & 178 & 18,112 & $1.28(1.10-1.48)$ \\
\hline Antifungals & D01 & 98 & 9650 & $1.26(1.03-1.54)$ \\
\hline Emollients and protectives & D02 & 4 & 144 & $3.41(1.26-9.26)$ \\
\hline Antipruritics, including antihistamines & D04 & 4 & 106 & $4.67(1.71-12.73)$ \\
\hline Antibacterials & D06 & 92 & 8639 & $1.24(1.01-1.52)$ \\
\hline Corticosteroids & D07 & 131 & 12,209 & $1.37(1.15-1.63)$ \\
\hline Genitourinary system and sex hormones & G & 48 & 3032 & $2.67(2.00-3.56)$ \\
\hline Androgens & G03B & 8 & 72 & $13.80(6.57-28-98)$ \\
\hline Estrogens & $\mathrm{G} 03 \mathrm{C}$ & 3 & 10 & $144.75(24.17-866.76)$ \\
\hline Antiandrogens & $\mathrm{G} 03 \mathrm{H}$ & 3 & 10 & $69.37(15.50-310.53)$ \\
\hline Urologicals & G04 & 36 & 2860 & $2.12(1.52-2.96)$ \\
\hline Urinary frequency and incontinence & G04BD & 5 & 338 & $2.87(1.18-6.99)$ \\
\hline Erection disorders & G04BE & 21 & 1895 & $1.70(1.10-2.62)$ \\
\hline Benign prostatic hypertrophy & G04C & 17 & 1117 & $2.81(1.73-4.55)$ \\
\hline Systemic hormones, excluding sex hormones and insulins & H & 53 & 4688 & $1.55(1.18-2.03)$ \\
\hline Corticosteroids, systemic & $\mathrm{HO2}$ & 46 & 3746 & $1.76(1.32-2.36)$ \\
\hline Anti-infectives for systemic use & J & 205 & 22,245 & $1.25(1.09-1.44)$ \\
\hline Antibacterials & J01 & 201 & 22,060 & $1.22(1.06-1.40)$ \\
\hline Antineoplastics and immunomodulating medication & $\mathbf{L}$ & $<3$ & 365 & $0.70(0.18-2.83)$ \\
\hline Muscular-skeletal system & M & 138 & 13,868 & $1.54(1.30-1.83)$ \\
\hline Anti-inflammatory and antirheumatic products & M01 & 135 & 13,615 & $1.54(1.30-1.83)$ \\
\hline Muscle relaxants & M03 & 16 & 1157 & $1.80(1.10-2.96)$ \\
\hline Nervous system & $\mathbf{N}$ & 138 & 11,470 & $1.96(1.66-2.33)$ \\
\hline Analgesics & N02 & 107 & 8305 & $2.00(1.65-2.42)$ \\
\hline Antiepileptics & N03 & 36 & 1444 & $3.52(2.51-4.93)$ \\
\hline Psycholeptics & N05 & 66 & 5261 & $1.77(1.38-2.26)$ \\
\hline Antipsychotics & N05A & 31 & 1594 & $2.57(1.79-3.70)$ \\
\hline Anxiolytics & NO5B & 36 & 2945 & $1.70(1.22-2.37)$ \\
\hline Sedatives & N05C & 34 & 3231 & $1.44(1.02-2.02)$ \\
\hline Psychoanaleptics & N06 & 55 & 4262 & $1.75(1.34-2.89)$ \\
\hline Antidepressants & N06A & 43 & 3940 & 1.47 (1.09-1.98) \\
\hline Psychostimulants (ADHD) & N06B & 19 & 499 & $4.39(2.77-6.95)$ \\
\hline
\end{tabular}


Table 3 continued

\begin{tabular}{|c|c|c|c|c|}
\hline Medication & ATC code & $47, X Y Y(n)$ & Controls $(n)$ & Hazard ratio $(95 \% \mathrm{Cl})$ \\
\hline Alcohol dependence & NOTBB & 14 & 865 & $2.06(1.21-3.50)$ \\
\hline Respiratory system & $\mathbf{R}$ & 165 & 14856 & $1.75(1.50-2.05)$ \\
\hline Decongestants and other nasal preparations for topical use & R01A & 68 & 5945 & $1.40(1.10-1.78)$ \\
\hline Obstructive lung disease & R03 & 106 & 7180 & $2.05(1.69-2.49)$ \\
\hline Antihistamines, systemic & R06 & 87 & 6219 & $1.76(1.43-2.18)$ \\
\hline Sensory organs & $\mathbf{S}$ & 154 & 15985 & $1.21(1.03-1.42)$ \\
\hline Antiglaucomas & SO1E & 7 & 332 & $3.38(1.59-7.19)$ \\
\hline Decongestants and antiallergics & S01G & 41 & 3333 & $1.46(1.07-1.99)$ \\
\hline Various & $\mathbf{T}$ & 5 & 382 & $1.49(0.62-3.61)$ \\
\hline
\end{tabular}

Hazard ratios (HRs) for chapters according to the Anatomical Therapeutic Chemical System (ACT) are shown in bold, whether significant or not. If significant, HRs of ACT blocks and specific medications (italic) within the chapters are shown as well. According to ethics regulations, the exact number of cases with a given prescription is not

the significant increased risk of vasodilator prescriptions observed in 47,XYY syndrome.

Diagnoses related to congenital malformations were significantly increased among 47,XYY males and when subdividing the diagnoses, there was a significant increased risk of malformations related to the circulatory, musculoskeletal, and the genitourinary system, here among a significantly increased risk of undescended testes. Approximately $6 \%$ of $47, \mathrm{XXY}$ males were recorded with undescended testes compared with $2 \%$ of controls, the latter in accordance with the frequency of undescended testes in the general male population. ${ }^{15}$ Adequate levels of androgens during fetal development are considered prerequisite for normal descent of the testes. ${ }^{15}$ The increased risk of undescended testes among 47,XYY may therefore very likely indicate that the presence of a supernumerary $\mathrm{Y}$ chromosome increases the risk of testicular impairment already during fetal life. However, Ratcliffe et al. observed no difference in testosterone levels when comparing 46,XY and $47, \mathrm{XYY}$ fetuses. ${ }^{16}$ No clear pattern of malformations related to the circulatory system was found, probably owing to the small number of cases, whereas for the musculoskeletal system, there was an increased risk of deformities of the feet. To date, there has been no evidence of an increased frequency of congenital malformations among 47,XYY males. ${ }^{17}$ However, Robinson et al. suggested an increased frequency of minor anomalies in 47,XYY syndrome. Among 42 newborn boys with $47, \mathrm{XYY}$ syndrome, eight were found to present with minor anomalies. The anomalies, however, varied widely and with no clear pattern. None presented with deformities of the feet, but two children were reported with undefined heart murmur and two were reported with inguinal hernia. ${ }^{17}$ Heart murmur could indicate presence of malformations related to the circulatory system and inguinal hernia could indicate an inguinal localization of the testes, thus, potentially substantiating results from the present study.
Sperm counts in males with $47, \mathrm{XYY}$ have been reported as ranging from normal numbers to total azoospermia, ${ }^{18}$ and 47 , XYY males seem more prevalent in infertile populations. ${ }^{10}$ Here, 47,XYY was associated with a markedly increased risk of being registered with a diagnosis of infertility. Considering males from the age of 20 years to end of follow-up, 14\% (29/ 205 ) of $47, \mathrm{XYY}$ males were recorded with a diagnosis of infertility. For comparison, this was only the case in $2 \%$ of controls. Previously, Stochholm et al. reported that males diagnosed with 47,XYY syndrome were less likely to cohabitate than sex- and age-matched general population controls, ${ }^{19}$ and one can therefore speculate whether the proportion of infertile 47,XYY males indeed is even larger than observed in the present study.

Flat foot and knee valgus are commonly described features associated with the 47,XYY syndrome. ${ }^{20,21}$ This was substantiated in the present study, as both were found to occur more frequently among 47,XYY males than among controls. Moreover, there was a significantly increased risk of myopia, astigmatism, and glaucoma among males with 47,XYY syndrome, which, to our knowledge, has not been systematically reported previously.

Sex chromosome aneuploidies may be associated to changes in size, shape, and structure of the teeth as well as with changes in mandibular and palatal dimensions, with the 47 , $\mathrm{XYY}$ syndrome being associated with increased tooth size as well as with lengthening of the mandibular arch and subsequent jaw protrusion, ${ }^{20,22}$ conceivably due to increased mitotic potential in the presence of the extra Y chromosome leading to increased cell division at various stages of development. ${ }^{23}$ The increased occurrence of tooth developmental disorders as well as of anomalies of jaw and tooth position among 47 ,XYY males in the present study was thus in line with previous reports. Dental caries and prescriptions of caries prophylactic agents were significantly increased among 47,XYY males as well. An increased use of medication that, as a side effect, reduces the saliva amount (e.g., 
antipsychotics and antidepressants) and thus in turn promote caries, is a likely explanation hereof. The reduced socioeconomic status associated to 47,XYY syndrome ${ }^{19}$ may impact negatively on tooth health as well. Interestingly, however, dental caries is also reported as being more frequent in Klinefelter syndrome, ${ }^{24}$ and a genetic disposition is therefore very likely. The reason for this is not clear, but expression of escape genes in triplicate, such as the AMELX, where variants are known to be involved in enamel hypoplasia, and the Y homolog AMELY, ${ }^{25}$ may be involved.

Records of pneumonia, asthma, and chronic obstructive lung disease were more frequent among 47,XYY males than among controls-a finding that was substantiated by 47,XYY males having an increased risk of prescriptions associated to obstructive lung disease. Previously, we and others reported on an association between 47,XYY syndrome and mortality due to pulmonary diseases, ${ }^{7,8}$ and other studies have reported that asthma seems to occur more frequently among $47, \mathrm{XYY}$ males as well. ${ }^{20}$ The underlying mechanisms have, however, not been accounted for. Unfortunately, in the present study, we were not able to account for smoking habits.

Varicose ulcerations, varicose veins, and venous ulcers have been reported in males affected by the 47,XYY syndrome, however, not systematically. ${ }^{21,26,27}$ Here, 47,XYY syndrome was associated with a significant increased risk of both leg varicose veins, ulcers of the lower limb, venous thrombophlebitis, and venous thromboembolism. Further, there was a significant increased risk of antithrombotic prescriptions among 47,XYY males. This may relate to hypogonadism and obesity, ${ }^{28}$ both observed with increased occurrence of among 47,XYY males in the present study. However, as a similar pattern is seen in Klinefelter syndrome, ${ }^{13}$ where we have previously shown an altered profile of the coagulation system, ${ }^{29}$ and it can be speculated whether the balance of the coagulation system is also altered in 47,XYY syndrome.

Chromosomal imbalances, perhaps especially supernumerary imbalances, are associated with an increased risk of seizures and epilepsy, ${ }^{30}$ and a possible link between seizures and the 47,XYY karyotype has previously been suggested, 7,20 likewise in Klinefelter syndrome. ${ }^{31}$ The present study supports this suggestion as there was an increased frequency of epilepsy among 47,XYY males along with an increased frequency of antiepileptic prescriptions. In addition, there was an increased frequency of febrile convulsions. A contributing factor to this increased seizure risk in 47,XYY syndrome may be explained by an abnormal cerebral morphology with increased gray and white matter ${ }^{32}$ that potentially may alter the synaptic function.

Psychiatric diagnoses seem more prevalent among 47,XYY males than among controls, ${ }^{20}$ and among 19 adolescent 47 , XYY males, Ratcliffe et al. reported that nearly half had been referred for psychiatric evaluation. ${ }^{33}$ This study confirms a pronounced psychiatric vulnerability in males affected by the $47, \mathrm{XYY}$ syndrome since an increased risk of both schizophrenia and bipolar disorders were observed in addition to an increased risk of having antidepressant prescribed. Further, there was an increased risk of mental or behavioral disorders due to use of alcohol or drugs, and medications used in the treatment of alcohol dependence were more frequently prescribed, thus supporting previous suggestions of an association between 47,XYY syndrome and an increased risk of alcoholism. ${ }^{34}$ Moreover, psychiatric disease is a well-known risk factor for alcohol or drug abuse and vice versa, and both may predispose to a poorer socioeconomic status as well as to an increased risk of criminal behavior, which, unfortunately, have shown to be the case for many men diagnosed with 47, XYY syndrome. ${ }^{19,35}$ We speculate whether earlier diagnosis and intervention in childhood with measures to increase school performance could alter this apparently vicious cycle.

Psychological developmental disorders, including autism spectrum disorders, have been observed more frequently in $47, \mathrm{XYY}$ syndrome than in controls, ${ }^{20,36}$ and general intelligence is typically reported as similar or mildly diminished, with verbal IQ being more diminished than performance IQ. ${ }^{4,20,33,36}$ The present study supports these findings, as 47 , XYY males had an increased risk of psychological developmental disorders as well as an increased risk of intellectual disability. Unfortunately, data do not allow us to conclude on the severity of intellectual disability.

Comparing the presented morbidity pattern in $47, \mathrm{XYY}$ syndrome with the morbidity pattern found in Klinefelter syndrome, ${ }^{31}$ it is obvious that these two patterns are very similar. Indeed, these two syndromes seem to have more similarities than differences as the mortality pattern and socioeconomic profile are very similar. ${ }^{19}$ Moreover, both 47, $\mathrm{XYY}$ and Klinefelter syndrome are unfortunately associated to an increased rate of criminality, which to a large extent is explained by the poorer socioeconomic profile, ${ }^{35}$ and both syndromes are associated with a negatively affected cognitive and behavioral phenotype including speech and language disorders, behavioral problems, and reduced motor skills and educational achievements. ${ }^{6}$ Suffice it to say, it seems as if the addition of an extra sex chromosome affects all aspects of life, regardless of it being an $\mathrm{X}$ or a $\mathrm{Y}$ chromosome. However, more research is needed to pinpoint specific differences between 47,XYY and Klinefelter syndrome, for example related to hypergonadotropic hypogonadism, which is almost obligate in relation to Klinefelter syndrome, but probably only present in some of the males with 47,XYY syndrome. Part of the similarity between 47,XYY and Klinefelter syndrome may be due to an overlapping dosage effect of genes residing in the pseudoautosomal regions on the $\mathrm{X}$ and $\mathrm{Y}$ chromosomes. Moreover, genomic hypermethylation has been shown in Klinefelter syndrome, ${ }^{37}$ and it can be speculated that the epigenetic landscape of 47,XYY syndrome resembles that found in Klinefelter syndrome, and thus could contribute to the similarities observed between the two syndromes. The $\mathrm{X}$ and $\mathrm{Y}$ chromosomes differ profoundly in size and content. Only about 40 genes are expressed on the $\mathrm{Y}$ chromosome, whereof 17 genes have homologs on the $\mathrm{X}$ chromosome, which contains more than 600 genes. ${ }^{38}$ The genes with $\mathrm{X}$ and $\mathrm{Y}$ homologs are involved in regulating other genes throughout 
the entire genome. ${ }^{38}$ In addition, 12 of the remaining genes on the $\mathrm{Y}$ chromosome have identical haplotypes on the $\mathrm{X}$ chromosome, and may thus also play a role. Most of the few genes expressed on the $\mathrm{Y}$ chromosome largely relate to spermatogenesis and will be expected to be expressed in duplicate, while all the genes in the pseudoautosomal region will be expected to be expressed in triplicate and as mentioned, the SHOX gene leads to increased height in 47, $\mathrm{XYY}{ }^{2}$ A recent study assessed the aggregate effect of common variation, quantified as polygenic scores, and demonstrated that diverse traits such as height were uniformly distributed across 47,XXX, 47,XXY, and 47,XYY syndromes, ${ }^{39}$ emphasizing not only that an extra SHOX gene increases height in supernumerary SCAs, ${ }^{2}$ but also that common and rare variants can affect phenotype. A recent study implies that $\mathrm{X}$ and $\mathrm{Y}$ chromosome homologs show similar expression, suggesting that they could explain some of the similarities between 47,XXY and 47,XYY syndromes. ${ }^{40}$

\section{Strengths and limitations}

The setting of the present study is a nationwide approach with establishment of the patient cohort being based on data from the DCCR, which can be considered complete. All 47,XYY males diagnosed in Denmark are included. This approach minimizes selection bias substantially as the only form of bias is the clinical initiative to perform a karyotype and thereby diagnose the SCA. In contrast, most other studies are limited to settings in referral hospitals, thus studying more selective patient cohorts. The generally high validity of data in the DNPR, due to its administrative and prospective collection of data, is another major strength of the study. Moreover, by including data of prescribed medications, we were able to detect and conclude more precisely on diseases that often do not lead to a hospital encounter as they are managed by general practitioners (e.g., hypertension, mild depression, and musculoskeletal disorders such as osteoarthritis), thus providing a more complete picture of morbidity among 47,XYY males. Other strengths are the close matching with controls, enabling a strong comparison with the general population, and the complete follow-up, which allows accounting for changes in specific outcomes over time.

A drawback of the present study is the fact that only $20 \%$ of the expected number of 47,XYY males are diagnosed, thus leaving a large unselected cohort of 47,XYY males undiagnosed, ${ }^{1}$ to whom results of the present study may not extend. Potentially, those who are undiagnosed may exhibit a completely normal phenotype despite the impression from general clinical observation. Only with the screening of a very large cohort or an entire population will this enigma be revealed. Nevertheless, those accounted for here correspond to the $47, \mathrm{XYY}$ population whom clinicians know from daily practice. Knowledge of specific genotype-phenotype associations are important in the clinical assessment of patients with genetic diseases. Owing to the low patient counts of 46,XY/47, XYY mosaicism and other karyotypes, we were, however, restricted from stratifying on the karyotype in our subanalyses of diagnoses and prescriptions. This must be taken into account when assessing and counseling patients with, e.g., mosaicism, as the degree of mosaicism, as well as the tissues affected by the abnormal genotype, are crucial for the phenotype and therefore the long-term prognosis.

\section{Conclusion}

We report a systematic investigation of morbidity in an unselected nationwide cohort of 47,XYY males using complete data on hospital diagnoses and prescribed medication. The emerging picture is a substantially increased comorbidity in 47,XYY syndrome, which is not confined to a single or a few diseases, but indeed to a wide variety of diseases. The study does not allow us to draw conclusions on the reasons for this increased morbidity. The supernumerary Y chromosome may be a direct effect of some of the diseases, whereas reduced health, likely because of poor socioeconomic status, may have an effect on other diseases. Increased awareness of the comorbidity risk profile among clinicians, in combination with a comprehensive multidisciplinary clinical assessment and counseling of 47,XYY males, may improve long-term health outcomes as well as socioeconomic profile in the 47,XYY syndrome. Further, it may improve counseling in case of prenatal detection of the 47,XYY karyotype, which should allow the parents to make a conscious and more rational decision about continuation of pregnancy.

\section{SUPPLEMENTARY INFORMATION}

The online version of this article (https://doi.org/10.1038/s41436020-0837-y) contains supplementary material, which is available to authorized users.

\section{ACKNOWLEDGEMENTS}

Data manager, Jan Hansen, is thanked for identifying the 47,XYY cohort in the DCCR. The study was financially supported by research grants from The Novo Nordisk Foundation (NNF13OC0003234, NNF15OC0016474) and the Familien Hede Nielsen Foundation. C.H.G., A.B., and K.S. are members of the European Reference Network on Rare Endocrine Conditions (ENDO-ERN), project ID number 739543.

\section{DISCLOSURE}

The authors declare no conflicts of interest.

Publisher's note Springer Nature remains neutral with regard to jurisdictional claims in published maps and institutional affiliations.

\section{REFERENCES}

1. Berglund A, Viuff MH, Skakkebaek A, Chang S, Stochholm K, Gravholt $\mathrm{CH}$. Changes in the cohort composition of turner syndrome and severe non-diagnosis of Klinefelter, 47,XXX and 47,XYY syndrome: a nationwide cohort study. Orphanet J Rare Dis. 2019;14:16.

2. Ottesen AM, Aksglaede L, Garn I, et al. Increased number of sex chromosomes affects height in a nonlinear fashion: a study of 305 patients with sex chromosome aneuploidy. Am J Med Genet A. 2010;152a:1206-1212. 
3. Jacobs PA, Brunton M, Melville MM, Brittain RP, McClemont WF. Aggressive behaviour, mental sub-normality and the XYY male. Nature. 1965;208:1351-1352.

4. Leggett V, Jacobs P, Nation K, Scerif G, Bishop DV. Neurocognitive outcomes of individuals with a sex chromosome trisomy: $X X X, X Y Y$, or XXY: a systematic review. Dev Med Child Neurol. 2010;52:119-129.

5. Lee NR, Wallace GL, Adeyemi El, et al. Dosage effects of $X$ and $Y$ chromosomes on language and social functioning in children with supernumerary sex chromosome aneuploidies: implications for idiopathic language impairment and autism spectrum disorders. J Child Psychol Psychiatry. 2012;53:1072-1081.

6. Ross JL, Roeltgen DP, Kushner $\mathrm{H}$, et al. Behavioral and social phenotypes in boys with $47, \mathrm{XYY}$ syndrome or $47, \mathrm{XXY}$ Klinefelter syndrome. Pediatrics. 2012;129:769-778.

7. Higgins CD, Swerdlow AJ, Schoemaker MJ, Wright AF, Jacobs PA, UK Clinical Cytogenetics Group. Mortality and cancer incidence in males with Y polysomy in Britain: a cohort study. Hum Genet. 2007;121:691-696.

8. Stochholm K, Juul S, Gravholt CH. Diagnosis and mortality in 47,XYY persons: a registry study. Orphanet J Rare Dis. 2010;5:15.

9. Schiavi RC, Theilgaard A, Owen DR, White D. Sex chromosome anomalies, hormones, and aggressivity. Arch Gen Psychiatry. 1984;41: 93-99.

10. El-Dahtory F, Elsheikha HM. Male infertility related to an aberrant karyotype, 47,XYY: four case reports. Cases J. 2009;2:28.

11. Price $W H$, van der Molen HJ. Plasma testosterone levels in males with the 47,XYY karyotype. J Endocrinol. 1970;47:117-122.

12. Gravholt $\mathrm{CH}$, Chang S, Wallentin M, Fedder J, Moore P, Skakkebaek A. Klinefelter syndrome: integrating genetics, neuropsychology, and endocrinology. Endocr Rev. 2018;39:389-423.

13. Chang S, Christiansen CF, Bojesen A, Juul S, Munster AB, Gravholt CH. Klinefelter syndrome and testosterone treatment: a national cohort study on thrombosis risk. Endocr Connect. 2019;9:34-43.

14. Mushannen T, Cortez P, Stanford FC, Singhal V. Obesity and hypogonadism - a narrative review highlighting the need for highquality data in adolescents. Children. 2019;6:63.

15. Toppari J, Kaleva M. Maldescendus testis. Horm Res. 1999;51:261-269.

16. Ratcliffe SG, Read G, Pan H, Fear C, Lindenbaum R, Crossley J. Prenatal testosterone levels in XXY and XYY males. Horm Res. 1994;42: 106-109.

17. Robinson A, Lubs HA, Nielsen J, Sorensen K. Summary of clinical findings: profiles of children with 47,XXY, 47,XXX and 47,XYY karyotypes. Birth Defects Orig Artic Ser. 1979;15:261-266.

18. Skakkebæk NE, Hultén M, Jacobsen P, Mikkelsen M. Quantification of human semineferous epithelium. Reproduction. 1973;32:391-401.

19. Stochholm K, Juul S, Gravholt $\mathrm{CH}$. Socio-economic factors affect mortality in 47,XYY syndrome-a comparison with the background population and Klinefelter syndrome. Am J Med Genet A. 2012;158a: 2421-2429.

20. Bardsley MZ, Kowal K, Levy C, et al. 47,XYY syndrome: clinical phenotype and timing of ascertainment. J Pediatr. 2013;163:1085-1094.

21. Parker CE, Melyk J, Fish CH. The XYY syndrome. Am J Med. 1969;47: 801-808.
22. Laine $T$, Alvesalo L. Palatal and mandibular arch morphology in $47, X Y Y$ men and in other sex-chromosome anomalies. Arch Oral Biol. 1993;38: 101-105.

23. Alvesalo L. Human sex chromosomes in oral and craniofacial growth. Arch Oral Biol. 2009;54:S18-S24.

24. Jorgensen IF, Russo F, Jensen $A B$, et al. Comorbidity landscape of the Danish patient population affected by chromosome abnormalities. Genet Med. 2019;21:2485-2495.

25. Hu JCC, Chan H-C, Simmer SG, et al. Amelogenesis imperfecta in two families with defined AMELX deletions in ARHGAP6. PLOS One. 2012;7: e52052-e52052.

26. Brown WM, Price $W H$, Jacobs $P A$. Further information on the identity of 47,XYY males. Br Med J. 1968;2:325-328.

27. Gattringer C, Scheurecker C, Hopfl R, Muller H. Association between venous leg ulcers and sex chromosome anomalies in men. Acta Derm Venereol. 2010;90:612-615.

28. Ruige JB, Ouwens DM, Kaufman J-M. Beneficial and adverse effects of testosterone on the cardiovascular system in men. J Clin Endocrinol Metab. 2013:98:4300-4310.

29. Chang S, Biltoft D, Skakkebaek A, et al. Testosterone treatment and association with thrombin generation and coagulation inhibition in Klinefelter syndrome: a cross-sectional study. Thromb Res. 2019;182:175-181.

30. Sorge G, Sorge A. Epilepsy and chromosomal abnormalities. Ital J Pediatr. 2010;36:36-36.

31. Bojesen A, Juul S, Birkebaek NH, Gravholt CH. Morbidity in Klinefelter syndrome: a Danish register study based on hospital discharge diagnoses. J Clin Endocrinol Metab. 2006;91:1254-1260.

32. Bryant DM, Hoeft F, Lai S, et al. Sex chromosomes and the brain: a study of neuroanatomy in XYY syndrome. Dev Med Child Neurol. 2012;54: 1149-1156.

33. Ratcliffe S. Long-term outcome in children of sex chromosome abnormalities. Arch Dis Child. 1999:80:192-195.

34. Mors O, Mortensen PB, Ewald $H$. No evidence of increased risk for schizophrenia or bipolar affective disorder in persons with aneuploidies of the sex chromosomes. Psychol Med. 2001;31:425-430.

35. Stochholm K, Bojesen A, Jensen AS, Juul S, Gravholt CH. Criminality in men with Klinefelter's syndrome and XYY syndrome: a cohort study. BMJ Open. 2012;2:e000650.

36. Geerts M, Steyaert J, Fryns JP. The XYY syndrome: a follow-up study on 38 boys. Genet Couns. 2003;14:267-279.

37. Skakkebaek A, Nielsen MM, Trolle C, et al. DNA hypermethylation and differential gene expression associated with Klinefelter syndrome. Sci Rep. 2018;8:13740.

38. Bellott DW, Hughes JF, Skaletsky H, et al. Mammalian Y chromosomes retain widely expressed dosage-sensitive regulators. Nature. 2014;508: 494-499.

39. Oetjens MT, Kelly MA, Sturm AC, Martin CL, Ledbetter DH. Quantifying the polygenic contribution to variable expressivity in eleven rare genetic disorders. Nat Commun. 2019;10:4897.

40. Raznahan A, Parikshak NN, Chandran V, et al. Sex-chromosome dosage effects on gene expression in humans. Proc Natl Acad Sci U S A. 2018;115:7398-7403. 